\title{
Nevada Test Site Groundwater Well Rehabilitation Plan
}

\section{December 2006}

Prepared for:

U. S. Department of Energy

National Nuclear Security Administration

Nevada Site Office

Prepared by:

Environmental Technical Services

National Security Technologies, LLC 
This Page Intentionally Left Blank 


\section{LIST OF ACRONYMS AND ABBREVIATIONS}

$\begin{array}{ll}\text { bgs } & \text { below ground surface } \\ \text { CAU } & \text { Corrective Action Unit } \\ \text { DC } & \text { direct current } \\ \text { ETS } & \text { Environmental Technical Services } \\ \mathrm{ft} & \text { feet } \\ \text { FY } & \text { fiscal year } \\ { }^{3} \mathrm{H} & \text { tritium } \\ \text { HSU } & \text { hydrostratigraphic unit } \\ \text { LCA } & \text { Lower Carbonate Aquifer } \\ \text { NAFR } & \text { Nellis Air Force Range } \\ \text { NSTec } & \text { National Security Technologies, LLC } \\ \text { NTS } & \text { Nevada Test Site } \\ \text { pCi/L } & \text { picoCurie per liter } \\ \text { ROM } & \text { rough order of magnitude }\end{array}$


This Page Intentionally Left Blank 


\subsection{PURPOSE}

This plan describes actions to improve the utility and credibility of the Nevada Test Site (NTS) interim groundwater monitoring program. The two principal actions are: 1) well maintenance/ rehabilitation activities and 2) the deployment of dedicated low-cost and reliable jack-pumps for groundwater sampling from deep monitoring wells. The scope of this proposal is to perform these actions on some number of nine selected wells (Figure 1) to evaluate whether these actions are achievable, practical, cost effective, and result in improved groundwater data quality.

\subsection{CONSTRAINTS ON ACHIEVING PROGRAM OBJECTIVES}

This plan has been developed to address potential credibility issues in the current groundwater monitoring program, principally the reliability of the groundwater chemistry data being generated. Most wells currently available to the groundwater monitoring program were not specifically designed and constructed for long-term groundwater quality monitoring purposes. Some wells may have vertically extensive completion zones and some wells have obstructions in them (e.g., pumps and tubing). Subsurface components of some wells include high-volume pumps that are unsuitable for long-term monitoring tasks. Purging with high-volume pumps results in extensive volumes of water that may have to be contained and characterized before subsequent disposition. The configuration of some wells necessitates the sampling of stagnant well water via wireline bailer. Although bailer sampling allows for the collection of depth-discrete samples, the collected samples may not be representative of local groundwater chemistry. To eliminate concerns about crosscontamination, the use of dedicated equipment is greatly preferred.

\subsection{PROPOSED RESOLUTIONS}

\subsection{Well Maintenance}

Water samples of questionable quality or unknown origin within the wellbore and concerns regarding the potential for cross-contamination within wells having extensive completions result in observed problems including:

- Recognizably unrepresentative samples (turbid and/or malodorous fluid);

- Anomalous water chemistry (elevated ions, poor charge balances); and

- Indeterminate interval of sample collection.

It is suggested that the following three fundamental activities be completed at each well evaluated:

1. Video logging to evaluate the condition of the casing, tubing, and borehole wall;

2. Chemistry logging to obtain current/pre-installation profile of water column; and

3. Reconditioning via mechanical and/or hydraulic stresses and fluid removal.

Should more substantial rehabilitation be necessary, the following ancillary activities (or others deemed more appropriate) may be conducted:

4. Isolating intervals with bridge-plugs/packers;

5. Accessing selected intervals/hydrostratigraphic units (HSUs) via perforation of blank casing; and/or

6. Deepening of a borehole. 


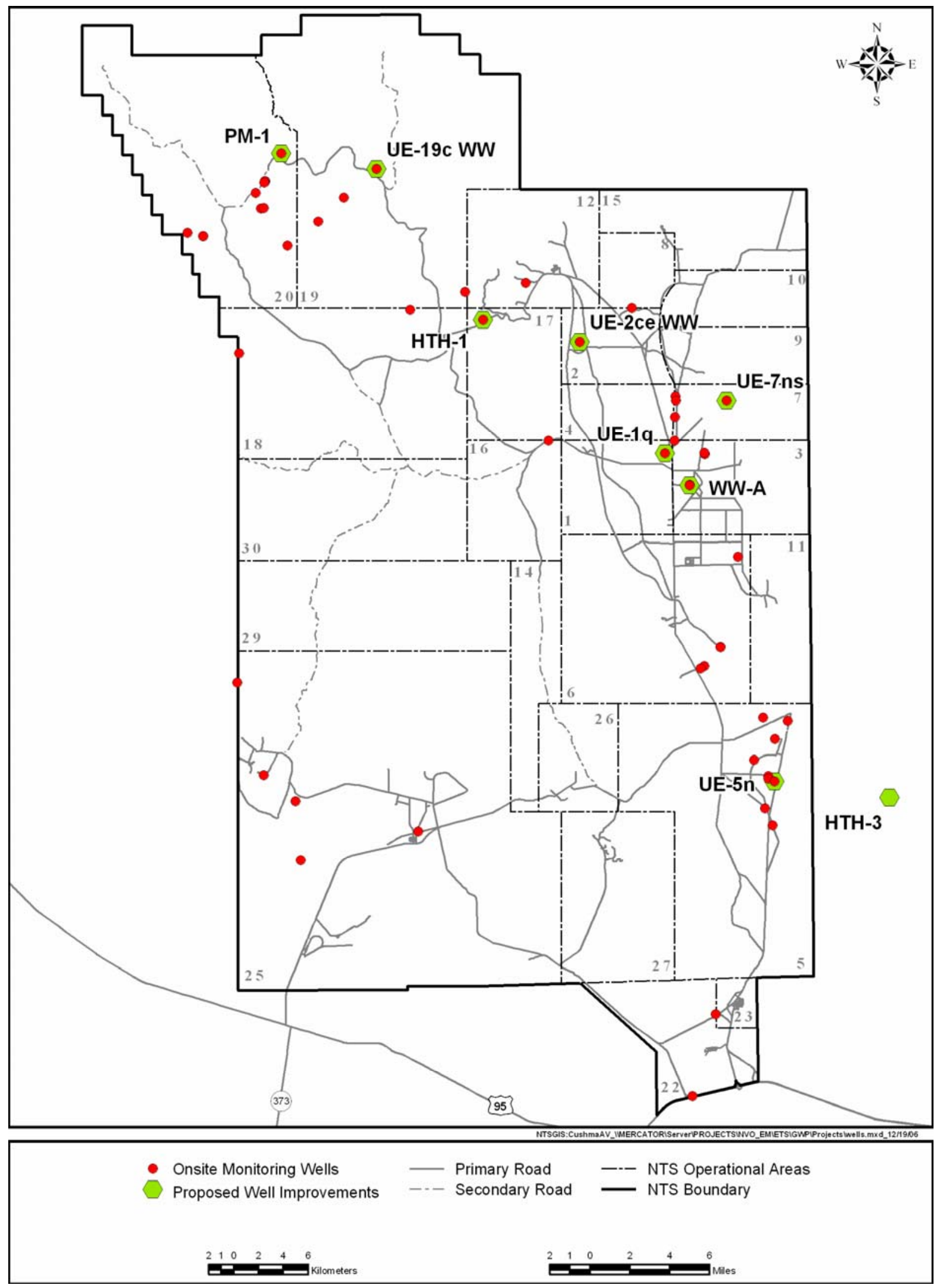

Figure 1. Wells Proposed for Improvements 


\subsection{Deployment of Jack-Pumps}

The National Security Technologies, LLC (NSTec), proposes to deploy low-maintenance jackpumps in selected monitoring wells. These pumps provide the lift capacity to produce groundwater from the deep aquifers encountered in the arid environment of the NTS without the considerable labor and electrical power requirements of electric submersible pumps. It is anticipated that direct current (DC) power can be supplied to DC motors powering the units principally by dedicated solarbased systems. Access tubing will be installed concurrent with the installation of the pump string to provide downhole access for water-level measurements or other wireline instruments. The actual set depths of the pumps will have to be considered on a well-specific basis (Note: The costs provided in this proposal are all based on pump set depths 200 feet [ft] below each well's static water level). Some criteria for determining the set depths of the pumps are:

- Intervals of slotted casing and stemmed filterpack;

- Hydraulic lift (total dynamic head);

- Water column above intake;

- Potential vertical gradients;

- Ability to monitor HSUs of interest; and

- Horizons of proximal sources/working points.

\subsubsection{Prioritization}

Nine candidate wells have been identified for possible deployment of the pumps (Table 1). These wells have been prioritized based on several criteria, including:

- History of detectable tritium $\left({ }^{3} \mathrm{H}\right)$ (increasing/decreasing trends);

- Completion zones (accessing HSUs of interest);

- Configuration (no downhole equipment and casing id); and

- Location (along different flowpaths, downgradient of sources, remote locations, and/or access limitations).

Table 1. Candidate Wells for Deployment of Jack-Pumps

\begin{tabular}{|c|l|c|c|l|}
\hline Priority & \multicolumn{1}{|c|}{ Well } & $\begin{array}{c}\text { Tritium Activity } \\
\text { (pCi/L) }\end{array}$ & $\begin{array}{c}\text { Water } \\
\text { Depth } \\
\text { (ft) }\end{array}$ & Comments \\
\hline 1 & UE-5n & 120,000 & 706 & $\begin{array}{l}\text { Increasing }{ }^{3} \text { H trend in alluvium; location is readily accessible } \\
\text { (pilot study/improvements) and visible (technology } \\
\text { demonstrations); ability to use grid power if needed }\end{array}$ \\
\hline 2 & UE-2ce WW & 120,000 & 1,448 & Testing-related radioactivity detected in the LCA \\
\hline 3 & Water Well A & 650 & 1,600 & Increasing ${ }^{3}$ H trend \\
\hline 4 & UE-7ns & 200 & 1,969 & Decreasing ${ }^{3}$ H trend \\
\hline 5 & PM-1 & 200 & 2,099 & One of the deepest wells on the NTS; remote location \\
\hline 6 & HTH \#3 & Non-detect & 1,104 & Only well completed in the LCA east of the NTS (on NAFR) \\
\hline 7 & HTH \#1 & Non-detect & 1,461 & Downgradient of Rainier Mesa CAU \\
\hline 8 & UE-1q & Non-detect & 1,655 & Completed in the LCA near a major fault (Topgallant) \\
\hline 9 & UE-19c WW & Non-detect & 2,339 & $\begin{array}{l}\text { Inactive non-potable water-supply well within Central Pahute } \\
\text { removed }\end{array}$ \\
\hline
\end{tabular}

pCi/L - picocuries per liter; LCA - Lower Carbonate Aquifer (regional carbonate aquifer underlying the NTS);

NAFR - Nellis Air Force Range; CAU - Corrective Action Unit 


\subsection{BUDGETS AND SCHEDULES}

The budget estimates include NSTec support for all phases of the proposed work (e.g., pre-field preparations, technical oversight of the field operations, and reporting/closeout). The budget and schedule estimates are rough-order-of-magnitude (ROM) estimates. Table 2 presents cost estimates for the field operations for each of the nine proposed wells. Table 3 presents cost and schedule estimates for the technical oversight of field operations for each of the nine wells. Table 4 presents the combined total estimated costs for field work and technical oversight by well. The work scope is scheduled to be completed in a single fiscal year (FY). A generic flow diagram of the proposed field operations is shown in Figure 2.

The total ROM cost estimates, by well, range from \$74,200 for Well UE-5n to \$169,350 for Well UE-19c WW (Table 4). UE-5n is the shallowest of the nine proposed wells (Table 1), and minimal well maintenance tasks are proposed. Pump installation at UE-5n is at a set depth of $905 \mathrm{ft}$ below ground surface (bgs), the borehole is unobstructed, and there is little need for well rehabilitation. UE-19c WW is the deepest of the nine wells (Table 1). The UE-19c estimate includes all of the work elements of the UE-5n estimate; it also includes additional work such as the removal of an existing pump, downhole tubing, and the hydraulic rehabilitation of the well. UE-19c WW has a pump set depth of 2,540 ft bgs.

For field operations of such magnitude, it is recognized that significant efficiencies are realized the more tasks are scheduled and completed over short durations. As such, the estimates were developed assuming full funding so all of the well maintenance and jack-pump installations could be completed in a single FY. Should the proposed tasks be funded over multiple FYs, additional support cost (e.g., recurring task planning and possible implementation of new programmatic requirements) can be expected. Additionally, costs for subcontracted work (e.g., pump installation and logging) may increase should multiple contracts, mobilizations, and/or training sessions be required over several FYs. If approved, NSTec will attempt to contract the work appropriately (e.g., staged contracting) to minimize extraneous costs to the client. 
Table 2. Estimated (Loaded) Costs for Field Operations

\begin{tabular}{|c|c|c|c|c|c|c|c|c|c|}
\hline \multirow{2}{*}{ Activity } & \multicolumn{3}{|c|}{ UE-5n } & \multicolumn{3}{|c|}{ UE-2c WW } & \multicolumn{3}{|c|}{ Water Well A } \\
\hline & ${ }^{1}$ Labor & ${ }^{2}$ Equipment & ${ }^{3}$ Materials & ${ }^{1}$ Labor & ${ }^{2}$ Equipment & ${ }^{3}$ Materials & ${ }^{1}$ Labor & ${ }^{2}$ Equipment & ${ }^{3}$ Materials \\
\hline $\begin{array}{l}\text { Mobilization/Demobilization } \\
\text { - Supply fuel, toilets, dumpster, } \\
\text { generator, forklift } \\
\text { - Drill anchor holes } \\
\text { - Rig up/down }\end{array}$ & $\$ 14,288$ & $\$ 2,503$ & $\$ 3,850$ & $\$ 14,288$ & $\$ 2,503$ & $\$ 7,700$ & $\$ 14,288$ & $\$ 2,503$ & $\$ 5,775$ \\
\hline $\begin{array}{l}\text { Workover (as needed) } \\
\text { - Video/chemistry logging } \\
\text { - Remove downhole goods } \\
\text { - Reconditioning (mechanical/hydraulic) } \\
\text { - Rehabilitation (bridge-plugs, } \\
\text { perforating) }\end{array}$ & $\$ 9,345$ & $\$ 2,002$ & $\$ 3,850$ & $\$ 15,654$ & $\$ 3,353$ & $\$ 5,775$ & $\$ 14,603$ & $\$ 3,128$ & $\$ 5,775$ \\
\hline $\begin{array}{l}\text { Downhole Installation } \\
\text { - Pump string (tubing) } \\
\text { - Plunger string (rods) } \\
\text { - Access line tubing }\end{array}$ & $\$ 5,423$ & $\$ 1,148$ & $\$ 52,500$ & $\$ 8,585$ & $\$ 1,817$ & $\$ 94,400$ & $\$ 9,159$ & $\$ 1,939$ & $\$ 102,000$ \\
\hline $\begin{array}{l}\text { Surficial Plumbing } \\
\text { - Flow lines/valves } \\
\text { - Stuffing box }\end{array}$ & $\$ 2,279$ & $\$ 0$ & $\$ 8,500$ & $\$ 2,279$ & $\$ 0$ & $\$ 8,500$ & $\$ 2,279$ & $\$ 0$ & $\$ 8,500$ \\
\hline $\begin{array}{l}\text { Unit Assembly and Testing } \\
\text { - Set pre-fabricated pad } \\
\text { - Set/assemble unit } \\
\text { - Electrical connections } \\
\text { - Test mechanical/electrical operation }\end{array}$ & $\$ 8,130$ & $\$ 500$ & $\$ 5,500$ & $\$ 8,130$ & $\$ 500$ & $\$ 5,500$ & $\$ 8,130$ & $\$ 500$ & $\$ 5,500$ \\
\hline Subtotal & $\$ 39,464$ & $\$ 6,153$ & $\$ 74,200$ & $\$ 48,935$ & $\$ 8,173$ & $\$ 119,950$ & $\$ 48,458$ & $\$ 8,070$ & $\$ 127,550$ \\
\hline Grand Total & \multicolumn{3}{|c|}{$\$ 119,817$} & \multicolumn{3}{|c|}{$\$ 177,058$} & \multicolumn{3}{|c|}{$\$ 184,078$} \\
\hline
\end{tabular}

${ }^{1}$ Based on man-hours for field personnel

${ }^{2}$ Includes rental costs for equipment used

${ }^{3}$ Expended supplies and dedicated goods 
Table 2. Estimated (Loaded) Costs for Field Operations (continued)

\begin{tabular}{|c|c|c|c|c|c|c|c|c|c|}
\hline \multirow{2}{*}{ Activity } & \multicolumn{3}{|c|}{ UE-7ns } & \multicolumn{3}{|c|}{ PM-1 } & \multicolumn{3}{|c|}{ НТН \#3 } \\
\hline & ${ }^{1}$ Labor & ${ }^{2}$ Equipment & ${ }^{3}$ Materials & ${ }^{1}$ Labor & ${ }^{2}$ Equipment & ${ }^{3}$ Materials & ${ }^{1}$ Labor & ${ }^{2}$ Equipment & ${ }^{3}$ Materials \\
\hline $\begin{array}{l}\text { Mobilization/Demobilization } \\
\text { - Supply fuel, toilets, dumpster, generator, } \\
\text { forklift } \\
\text { - Drill anchor holes } \\
\text { - Rig up/down }\end{array}$ & $\$ 14,288$ & $\$ 2,503$ & $\$ 7,700$ & $\$ 14,288$ & $\$ 2,503$ & $\$ 7,700$ & $\$ 14,288$ & $\$ 2,503$ & $\$ 3,750$ \\
\hline $\begin{array}{l}\text { Workover (as needed) } \\
\text { - Video/chemistry logging } \\
\text { - Remove downhole goods } \\
\text { - Reconditioning (mechanical/hydraulic) } \\
\text { - Rehabilitation (bridge-plugs, } \\
\text { perforating) }\end{array}$ & $\$ 15,933$ & $\$ 3,413$ & $\$ 7,700$ & $\$ 9,345$ & $\$ 2,002$ & $\$ 7,700$ & $\$ 12,462$ & $\$ 2,670$ & $\$ 3,750$ \\
\hline $\begin{array}{l}\text { Downhole Installation } \\
\text { - Pump string (tubing) } \\
\text { - Plunger string (rods) } \\
\text { - Access line tubing }\end{array}$ & $\$ 10,551$ & $\$ 2,234$ & $\$ 120,450$ & $\$ 12,198$ & $\$ 2,582$ & $\$ 142,250$ & $\$ 7,287$ & $\$ 1,542$ & $\$ 77,200$ \\
\hline $\begin{array}{l}\text { Surficial Plumbing } \\
\text { - Flow lines/valves } \\
\text { - Stuffing box }\end{array}$ & $\$ 2,279$ & $\$ 0$ & $\$ 8,500$ & $\$ 2,279$ & $\$ 0$ & $\$ 8,500$ & $\$ 2,279$ & $\$ 0$ & $\$ 8,500$ \\
\hline $\begin{array}{l}\text { Unit Assembly and Testing } \\
\text { - Set pre-fabricated pad } \\
\text { - Set/assemble unit } \\
\text { - Electrical connections } \\
\text { - Test mechanical/electrical operation }\end{array}$ & $\$ 8,130$ & $\$ 500$ & $\$ 5,500$ & $\$ 8,130$ & $\$ 500$ & $\$ 5,500$ & $\$ 8,130$ & $\$ 500$ & $\$ 6,000$ \\
\hline Subtotal & $\$ 51,180$ & $\$ 8,650$ & $\$ 149,850$ & $\$ 46,239$ & $\$ 7,587$ & $\$ 171,650$ & $\$ 44,445$ & $\$ 7,215$ & $\$ 99,200$ \\
\hline Grand Total & \multicolumn{3}{|c|}{$\$ 209,680$} & \multicolumn{3}{|c|}{$\$ 225,476$} & \multicolumn{3}{|c|}{$\$ 150,860$} \\
\hline
\end{tabular}

Based on man-hours for field personnel

Includes rental costs for equipment used

${ }^{3}$ Expended supplies and dedicated goods 
Table 2. Estimated (Loaded) Costs for Field Operations (continued)

\begin{tabular}{|c|c|c|c|c|c|c|c|c|c|}
\hline \multirow{2}{*}{ Activity } & \multicolumn{3}{|c|}{ HTH \#1 } & \multicolumn{3}{|c|}{ UE-1q } & \multicolumn{3}{|c|}{ UE-19c WW } \\
\hline & ${ }^{1}$ Labor & ${ }^{2}$ Equipment & ${ }^{3}$ Materials & ${ }^{1}$ Labor & ${ }^{2}$ Equipment & ${ }^{3}$ Materials & ${ }^{1}$ Labor & ${ }^{2}$ Equipment & ${ }^{3}$ Materials \\
\hline $\begin{array}{l}\text { Mobilization/Demobilization } \\
\text { - Supply fuel, toilets, dumpster, generator, } \\
\text { forklift } \\
\text { - Drill anchor holes } \\
\text { - Rig up/down }\end{array}$ & $\$ 14,288$ & $\$ 2,503$ & $\$ 7,700$ & $\$ 14,288$ & $\$ 2,503$ & $\$ 7,700$ & $\$ 14,288$ & $\$ 2,503$ & $\$ 7,700$ \\
\hline $\begin{array}{l}\text { Workover (as needed) } \\
\text { - Video/chemistry logging } \\
\text { - Remove downhole goods } \\
\text { - Reconditioning (mechanical/hydraulic) } \\
\text { - Rehabilitation (bridge-plugs, } \\
\text { perforating) }\end{array}$ & $\$ 9,345$ & $\$ 2,002$ & $\$ 7,700$ & $\$ 9,345$ & $\$ 2,002$ & $\$ 7,700$ & $\$ 18,023$ & $\$ 3,861$ & $\$ 7,700$ \\
\hline $\begin{array}{l}\text { Downhole Installation } \\
\text { - Pump string (tubing) } \\
\text { - Plunger string (rods) } \\
\text { - Access line tubing }\end{array}$ & $\$ 8,634$ & $\$ 1,828$ & $\$ 95,050$ & $\$ 9,366$ & $\$ 1,983$ & $\$ 104,750$ & $\$ 11,949$ & $\$ 2,529$ & $\$ 138,950$ \\
\hline $\begin{array}{l}\text { Surficial Plumbing } \\
\text { - Flow lines/valves } \\
\text { - Stuffing box }\end{array}$ & $\$ 2,279$ & $\$ 0$ & $\$ 8,500$ & $\$ 2,279$ & $\$ 0$ & $\$ 8,500$ & $\$ 2,279$ & $\$ 0$ & $\$ 8,500$ \\
\hline $\begin{array}{l}\text { Unit Assembly and Testing } \\
\text { - Set pre-fabricated pad } \\
\text { - Set/assemble unit } \\
\text { - Electrical connections } \\
\text { - Test mechanical/electrical operation }\end{array}$ & $\$ 8,130$ & $\$ 500$ & $\$ 5,500$ & $\$ 8,130$ & $\$ 500$ & $\$ 5,500$ & $\$ 8,130$ & $\$ 500$ & $\$ 5,500$ \\
\hline Subtotal & $\$ 42,675$ & $\$ 6,833$ & $\$ 124,450$ & $\$ 43,407$ & $\$ 6,988$ & $\$ 134,150$ & $\$ 54,668$ & $\$ 9,393$ & $\$ 168,350$ \\
\hline Grand Total & & $\$ 173,958$ & & & $\$ 184,545$ & & & $\$ 232,411$ & \\
\hline
\end{tabular}

Based on man-hours for field personnel

${ }^{2}$ Includes rental costs for equipment used

${ }^{3}$ Expended supplies and dedicated goods 
Table 3. Estimated Costs and Schedule for Technical Oversight

\begin{tabular}{|c|c|c|c|c|c|c|c|}
\hline Task & $\begin{array}{c}\text { Duration } \\
\text { (Working } \\
\text { Days) }^{1}\end{array}$ & Man-Hours & $\begin{array}{c}\text { ETS }^{2} \\
\text { Manager }\end{array}$ & $\begin{array}{c}\text { Senior } \\
\text { Scientist }\end{array}$ & Scientist & $\begin{array}{l}\text { Support } \\
\text { Staff }^{3}\end{array}$ & Cost \\
\hline \multicolumn{8}{|c|}{ Project Development and Completion } \\
\hline Plans / Documents & 12 & 200 & 20 & 80 & 40 & 60 & \\
\hline Contracting / Solicitation & 80 & 140 & 40 & 100 & 0 & 0 & \\
\hline Review / Award & 8 & 120 & 40 & 80 & 0 & 0 & \\
\hline Readiness / Pre-field & 8 & 110 & 20 & 40 & 20 & 30 & \\
\hline Reporting / Closeout & 24 & 230 & 40 & 120 & 40 & 30 & \\
\hline Subtotal & 132 & 800 & 160 & 420 & 100 & 120 & $\$ 48,900$ \\
\hline \multicolumn{8}{|c|}{ Technical Oversight for Field Operations - UE-5n } \\
\hline $\begin{array}{l}\text { Mobilization / } \\
\text { Demobilization }\end{array}$ & 2.5 & 50 & 10 & 30 & 10 & 0 & \\
\hline Workover & 2 & 30 & 10 & 20 & 0 & 0 & \\
\hline Installation & 1.5 & 50 & 10 & 20 & 0 & 20 & \\
\hline Surface Connections & 1 & 20 & 10 & 10 & 0 & 0 & \\
\hline Set and Test & 2 & 50 & 10 & 20 & 10 & 10 & \\
\hline Subtotal & 9 & 200 & 50 & 100 & 20 & 30 & $\$ 12,450$ \\
\hline \multicolumn{8}{|c|}{ Technical Oversight for Field Operations - UE-2c WW } \\
\hline $\begin{array}{l}\text { Mobilization / } \\
\text { Demobilization }\end{array}$ & 2.5 & 50 & 10 & 30 & 10 & 0 & \\
\hline Workover & 2 & 30 & 10 & 20 & 0 & 0 & \\
\hline Installation & 1.5 & 50 & 10 & 20 & 0 & 20 & \\
\hline Surface Connections & 2 & 20 & 10 & 10 & 0 & 0 & \\
\hline Set and Test & 3 & 50 & 10 & 20 & 10 & 10 & \\
\hline Subtotal & 11 & 200 & 50 & 100 & 20 & 30 & $\$ 12,450$ \\
\hline \multicolumn{8}{|c|}{ Technical Oversight for Field Operations - Water Well A } \\
\hline $\begin{array}{l}\text { Mobilization / } \\
\text { Demobilization }\end{array}$ & 2 & 50 & 10 & 30 & 10 & 0 & \\
\hline Workover & 3 & 30 & 10 & 20 & 0 & 0 & \\
\hline Installation & 2 & 60 & 10 & 20 & 10 & 20 & \\
\hline Surface Connections & 2 & 20 & 10 & 10 & 0 & 0 & \\
\hline Set and Test & 2 & 50 & 10 & 20 & 10 & 10 & \\
\hline Subtotal & 11 & 210 & 50 & 100 & 30 & 30 & $\$ 12,900$ \\
\hline \multicolumn{8}{|c|}{ Technical Oversight for Field Operations - UE-7ns } \\
\hline $\begin{array}{l}\text { Mobilization / } \\
\text { Demobilization }\end{array}$ & 2.5 & 50 & 10 & 30 & 10 & 0 & \\
\hline Workover & 4 & 30 & 10 & 20 & 0 & 0 & \\
\hline Installation & 2 & 50 & 10 & 20 & 0 & 20 & \\
\hline Surface Connections & 1 & 20 & 10 & 10 & 0 & 0 & \\
\hline Set and Test & 2 & 50 & 10 & 20 & 10 & 10 & \\
\hline Subtotal & 11.5 & 200 & 50 & 100 & 20 & 30 & $\$ 12,450$ \\
\hline \multicolumn{8}{|c|}{ Technical Oversight for Field Operations - PM-1 } \\
\hline $\begin{array}{l}\text { Mobilization / } \\
\text { Demobilization }\end{array}$ & 2.5 & 50 & 10 & 30 & 10 & 0 & \\
\hline Workover & 3 & 50 & 10 & 40 & 0 & 0 & \\
\hline Installation & 2 & 70 & 10 & 30 & 10 & 20 & \\
\hline Surface Connections & 1 & 20 & 10 & 10 & 0 & 0 & \\
\hline Set and Test & 2 & 50 & 10 & 20 & 10 & 10 & \\
\hline Subtotal & 10.5 & 240 & 50 & 130 & 30 & 30 & $\$ 14,700$ \\
\hline
\end{tabular}

${ }^{1}$ Based on a linear schedule of four 10-hour project days per week

${ }^{2}$ ETS - Environmental Technical Services

${ }^{3}$ Includes personnel from Health Physics, Health and Safety, and Waste Management 
Table 3. Estimated Costs and Schedule for Technical Oversight (continued)

\begin{tabular}{|c|c|c|c|c|c|c|c|}
\hline Task & $\begin{array}{c}\text { Duration } \\
\text { (Working } \\
\text { Days) }\end{array}$ & Man-Hours & $\begin{array}{c}\text { ETS }^{2} \\
\text { Manager }\end{array}$ & $\begin{array}{l}\text { Senior } \\
\text { Scientist }\end{array}$ & Scientist & $\begin{array}{l}\text { Support } \\
\text { Staff }^{3}\end{array}$ & Cost \\
\hline \multicolumn{8}{|c|}{ Technical Oversight for Field Operations - HTH \#3 } \\
\hline $\begin{array}{l}\text { Mobilization / } \\
\text { Demobilization }\end{array}$ & 2.5 & 50 & 10 & 30 & 10 & 0 & \\
\hline Workover & 3 & 30 & 10 & 20 & 0 & 0 & \\
\hline Installation & 2 & 50 & 10 & 20 & 0 & 20 & \\
\hline Surface Connections & 1 & 20 & 10 & 10 & 0 & 0 & \\
\hline Set and Test & 2 & 50 & 10 & 20 & 10 & 10 & \\
\hline Subtotal & 10.5 & 200 & 50 & 100 & 20 & 30 & $\$ 12,450$ \\
\hline \multicolumn{8}{|c|}{ Technical Oversight for Field Operations - HTH \#1 } \\
\hline $\begin{array}{l}\text { Mobilization / } \\
\text { Demobilization }\end{array}$ & 2.5 & 50 & 10 & 30 & 10 & 0 & \\
\hline Workover & 2 & 30 & 10 & 20 & 0 & 0 & \\
\hline Installation & 2 & 70 & 10 & 30 & 10 & 20 & \\
\hline Surface Connections & 1 & 20 & 10 & 10 & 0 & 0 & \\
\hline Set and Test & 2 & 50 & 10 & 20 & 10 & 10 & \\
\hline Subtotal & 9.5 & 220 & 50 & 110 & 30 & 30 & $\$ 13,500$ \\
\hline \multicolumn{8}{|c|}{ Technical Oversight for Field Operations - UE-1q } \\
\hline $\begin{array}{l}\text { Mobilization / } \\
\text { Demobilization }\end{array}$ & 2.5 & 50 & 10 & 30 & 10 & 0 & \\
\hline Workover & 2 & 30 & 10 & 20 & 0 & 0 & \\
\hline Installation & 2 & 50 & 10 & 20 & 0 & 20 & \\
\hline Surface Connections & 1 & 20 & 10 & 10 & 0 & 0 & \\
\hline Set and Test & 2.5 & 50 & 10 & 20 & 10 & 10 & \\
\hline Subtotal & 10 & 200 & 50 & 100 & 20 & 30 & $\$ 12,450$ \\
\hline \multicolumn{8}{|c|}{ Technical Oversight for Field Operations - UE-19c } \\
\hline $\begin{array}{l}\text { Mobilization / } \\
\text { Demobilization }\end{array}$ & 2.5 & 50 & 10 & 30 & 10 & 0 & \\
\hline Workover & 4 & 50 & 10 & 40 & 0 & 0 & \\
\hline Installation & 3 & 70 & 10 & 30 & 10 & 20 & \\
\hline Surface Connections & 1 & 20 & 10 & 10 & 0 & 0 & \\
\hline Set and Test & 2 & 50 & 10 & 20 & 10 & 10 & \\
\hline Subtotal & 12.5 & 240 & 50 & 130 & 30 & 30 & $\$ 14,700$ \\
\hline \multicolumn{7}{|c|}{ Grand Total } & $\$ 166,950$ \\
\hline
\end{tabular}

${ }^{1}$ Based on a linear schedule of four 10-hour project days per week

${ }^{2}$ ETS - Environmental Technical Services

${ }^{3}$ Includes personnel from Health Physics, Health and Safety, and Waste Management 
Table 4. Estimated Schedule and Cost for Nine Wells

\begin{tabular}{|c|c|c|c|c|c|}
\hline \multirow{2}{*}{ Task / Well } & \multirow{2}{*}{$\begin{array}{c}\text { Duration } \\
\text { (Work Days) }\end{array}$} & \multirow{2}{*}{$\begin{array}{c}\text { ETS Personnel and } \\
\text { Support Group Staffing }\end{array}$} & \multicolumn{3}{|c|}{ Field Operations } \\
\hline & & & Labor & Equipment & Materials \\
\hline Project Development & 132 & $\$ 48,900$ & --- & --- & --- \\
\hline UE-5n & 9 & $\$ 12,450$ & $\$ 39,464$ & $\$ 6,153$ & $\$ 74,200$ \\
\hline UE-2c WW & 11 & $\$ 12,450$ & $\$ 48,935$ & $\$ 8,173$ & $\$ 119,950$ \\
\hline Water Well A & 11 & $\$ 12,900$ & $\$ 48,458$ & $\$ 8,070$ & $\$ 127,550$ \\
\hline UE-7ns & 11.5 & $\$ 12,450$ & $\$ 51,180$ & $\$ 8,650$ & $\$ 149,850$ \\
\hline PM-1 & 10.5 & $\$ 14,700$ & $\$ 46,239$ & $\$ 7,587$ & $\$ 171,650$ \\
\hline НTH \#3 & 10.5 & $\$ 12,450$ & $\$ 44,445$ & $\$ 7,215$ & $\$ 99,200$ \\
\hline НТН \#1 & 9.5 & $\$ 13,500$ & $\$ 42,675$ & $\$ 6,833$ & $\$ 124,450$ \\
\hline UE-1q & 10 & $\$ 12,450$ & $\$ 43,407$ & $\$ 6,988$ & $\$ 134,150$ \\
\hline UE-19c WW & 12.5 & $\$ 14,700$ & $\$ 54,668$ & $\$ 9,393$ & $\$ 169,350$ \\
\hline Subtotal & 227.5 & $\$ 166,950$ & $\$ 419,471$ & $\$ 69,062$ & $\$ 1,170,350$ \\
\hline & & & & Grand Total & $\$ 1,337,300$ \\
\hline
\end{tabular}

Costs are loaded and assume full funding for the completion of work within a single FY. 


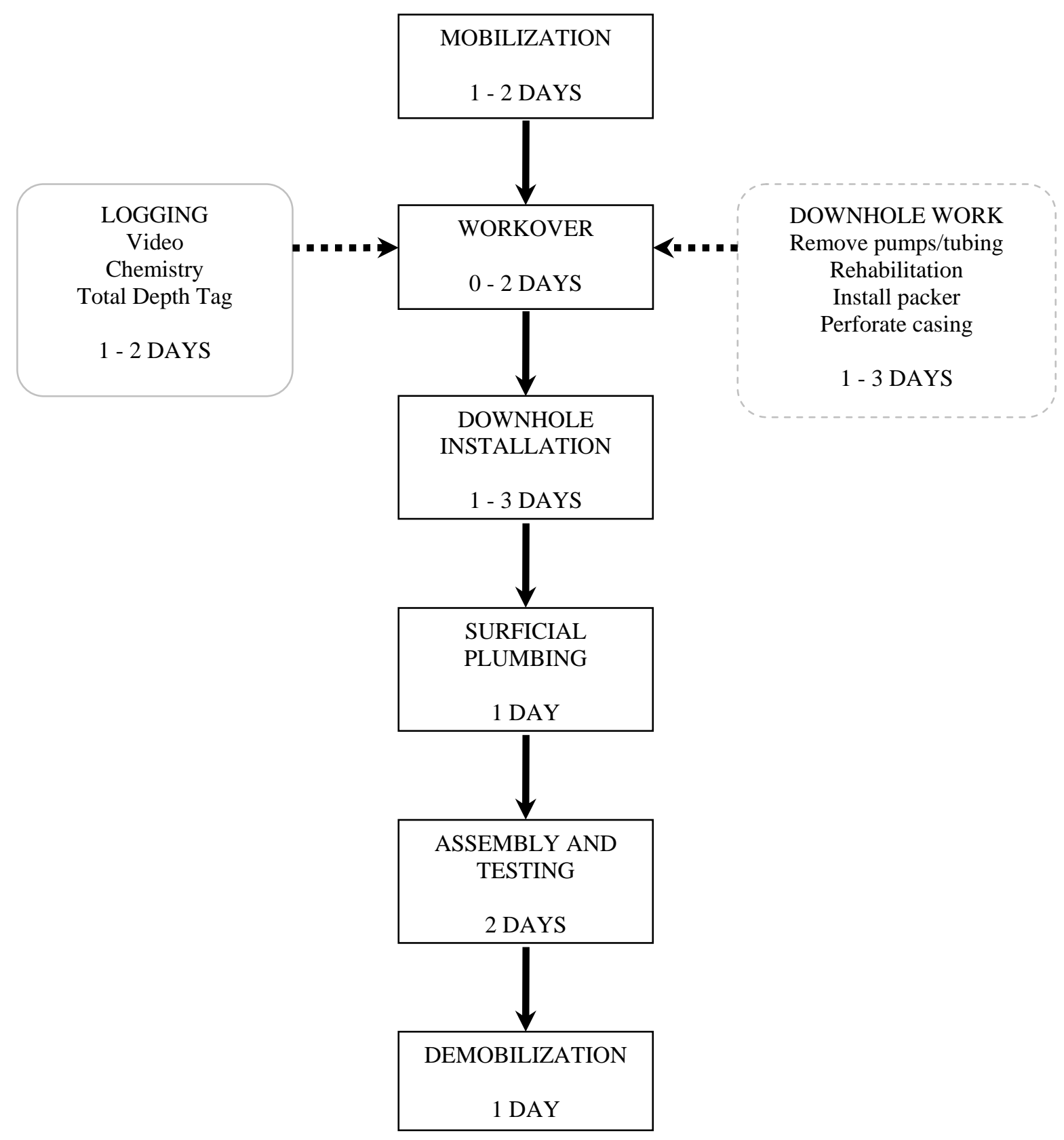

Figure 2. Generic Schedule of the Proposed Field Operations 
This Page Intentionally Left Blank 